\title{
THE POWER COLLECTION METHOD FOR CONNECTION RELATIONS: MEIXNER POLYNOMIALS
}

\author{
Michael A. Baeder, Howard S. Cohl, Roberto S. Costas-Santos \\ AND WENQING XU
}

Abstract. We introduce the power collection method for easily deriving connection relations for certain hypergeometric orthogonal polynomials in the $(q$-)Askey scheme. We summarize the full-extent to which the power collection method may be used. As an example, we use the power collection method to derive connection and connection-type relations for Meixner and Krawtchouk polynomials. These relations are then used to derive generalizations of generating functions for these orthogonal polynomials. The coefficients of these generalized generating functions are in general, given in terms of multiple hypergeometric functions. From derived generalized generating functions, we deduce corresponding contour integral and infinite series expressions by using orthogonality.

Mathematics subject classification (2010): 33C45, 05A15, 33C20, 34L10, 30E20.

Keywords and phrases: Generating functions, connection coefficients, connection-type relations, eigenfunction expansions, definite integrals, infinite series.

\section{REFERENCES}

[1] R. As Key, Orthogonal Polynomials and Special Functions, Society for Industrial and Applied Mathematics, Philadelphia, PA, 1975.

[2] M. A. BAEDER, H. S. COHL, AND H. VolKmer, Generalizations of generating functions for higher continuous hypergeometric orthogonal polynomials in the Askey scheme, Journal of Mathematical Analysis and Applications, 427 (1), 2015.

[3] H. Chaggara And W. Koepf, Duplication coefficients via generating functions, Complex Variables and Elliptic Equations. An International Journal, 52 (6): 537-549, 2007.

[4] H. S. Cohl, R. S. Costas-Santos, AND P. R. Hwang, Generalizations of generating functions for basic hypergeometric orthogonal polynomials, submitted, 2016.

[5] H. S. COHL, C. MACKenzie, And H. VolkMer, Generalizations of generating functions for hypergeometric orthogonal polynomials with definite integrals, Journal of Mathematical Analysis and Applications, 407 (2): 211-225, 2013.

[6] R. S. COSTAS-SANTOS AND J. F. SÁNCHEZ-LARA, Extensions of discrete classical orthogonal polynomials beyond the orthogonality, Journal of Computational and Applied Mathematics, 225 (2): 440451, 2009.

[7] F. W. J. Olver, A. B. Olde Daalhuis, D. W. Lozier, B. I. Schneider, R. F. Boisvert, C. W. Clark, B. R. Miller and B. V. Saunders, eds. NIST Digital Library of Mathematical Functions, http://dlmf .nist.gov/, Release 1.0.13 of 2016-09-16.

[8] M. Foupouagnigni, W. Koepf, And D. D. Tcheutia, Connection and linearization coefficients of the Askey-Wilson polynomials, Journal of Symbolic Computation, 53: 96-118, 2013.

[9] M. Foupouagnigni, W. Koepf, D. D. Tcheutia, and P. Nuionou Sadjang, Representations of q-orthogonal polynomials, Journal of Symbolic Computation, 47 (11): 1347-1371, 2012.

[10] G. GASPER, Projection formulas for orthogonal polynomials of a discrete variable, Journal of Mathematical Analysis and Applications, 45: 176-198, 1974. 
[11] M. E. H. Ismail, Classical and Quantum Orthogonal Polynomials in One Variable, volume 98 of Encyclopedia of Mathematics and its Applications, Cambridge University Press, Cambridge, 2005, with two chapters by Walter Van Assche.

[12] M. E. H. Ismail AND M. Rahman, Connection relations and expansions, Pacific Journal of Mathematics, 252 (2): 427-446, 2011.

[13] M. E. H. ISMAIL AND P. SimeONOv, Formulas and identities involving the Askey-Wilson operator, Advances in Applied Mathematics, 76: 68-96, 2016.

[14] M. E. H. Ismail AND D. Stanton, Expansions in the Askey-Wilson polynomials, Journal of Mathematical Analysis and Applications, 424 (1):664-674, 2015.

[15] R. KOEKOEK, P. A. LeSky, AND R. F. SwartTouw, Hypergeometric orthogonal polynomials and their q-analogues, Springer Monographs in Mathematics, Springer-Verlag, Berlin, 2010, with a foreword by Tom H. Koornwinder.

[16] W. Koepf AND D. Schmersau, Representations of orthogonal polynomials, Journal of Computational and Applied Mathematics, 90 (1): 57-94, 1998.

[17] E. D. RainVille, Special Functions, The Macmillan Co., New York, 1960.

[18] H. M. SRivastava ANd Per W. Karlsson, Multiple Gaussian hypergeometric series, Ellis Horwood Series: Mathematics and its Applications, Ellis Horwood Ltd., Chichester, 1985.

[19] D. D. Tcheutia, On Connection, Linearization and Duplication Coefficients of Classical Orthogonal Polynomials, $\mathrm{PhD}$ thesis, Universität Kassel, Kassel, Germany, 2014, viii+145 pages.

[20] D. D. Tcheutia, M. Foupouagnigni, W. Koepf, and P. Nuionou Sadjang, Coefficients of multiplication formulas for classical orthogonal polynomials, Ramanujan Journal. An International Journal Devoted to the Areas of Mathematics Influenced by Ramanujan, 39 (3): 497-531, 2016.

[21] N. M. Temme, Special Functions: an Introduction to the Classical Functions of Mathematical Physics, J. Wiley \& Sons, New York, 1996. 